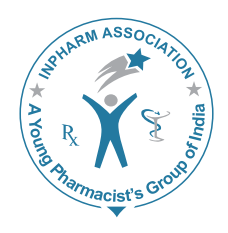

\title{
Sepbox Technique in Natural Products
}

\author{
Bhandari M, Bhandari Anil, Bhandari A ${ }^{1}$ \\ Departments of Pharmacognosy, Jodhpur College of Pharmacy, Jodhpur National University, Jodhpur, \\ ${ }^{1}$ Lachoo Memorial College of Science and Technology, Jodhpur, Rajasthan, India
}

Address for correspondence: Ms. Monika Bhandari; E-mail: monikabhandari85@yahoo.com

\begin{abstract}
Natural compounds still play an increasingly important role in many areas of research and development. Their structural diversity is enhanced by the synthetic potential of combinatorial chemistry. Fractionation and separation of samples obtained from nature remain time-consuming, tedious and extremely expensive even though the assays for testing these samples have become faster and more cost-effective thanks to advanced high-throughput screening (HTS) processes. Today, sepbox is the standard technology used for separating compounds from natural resources. Fractionations and separation of samples obtained from nature remain very difficult. Automation of sample preparation therefore has great economic potential. The unique sepbox concept allows processing sample automatically and will make up to 30 times faster than by using a conventional process.
\end{abstract}

Key words: High-throughput screening, natural compounds, separation, sepbox

\section{INTRODUCTION}

The sepbox concept is based on a patented combination of high-performance liquid chromatography (HPLC) and solidphase extraction (SPE) that provides a universal platform suitable for processing large sample numbers. This facility is commonly called as sepbox or the separation box shown in Figure 1. The facility is being used for the fractionation of plant extracts or pre-fractionated extracts and combinatorial reaction products. Using two-dimensional separations the recovery rate for both polar and non-polar substances is usually above $90 \%$ per cent. Using an automated and highly

\begin{tabular}{|l|l|}
\hline \multicolumn{2}{|c|}{ Access this article online } \\
\hline Quick Response Code: & \\
\hline & Website: \\
\hline & www.jyoungpharm.in \\
& DOI: \\
\hline
\end{tabular}

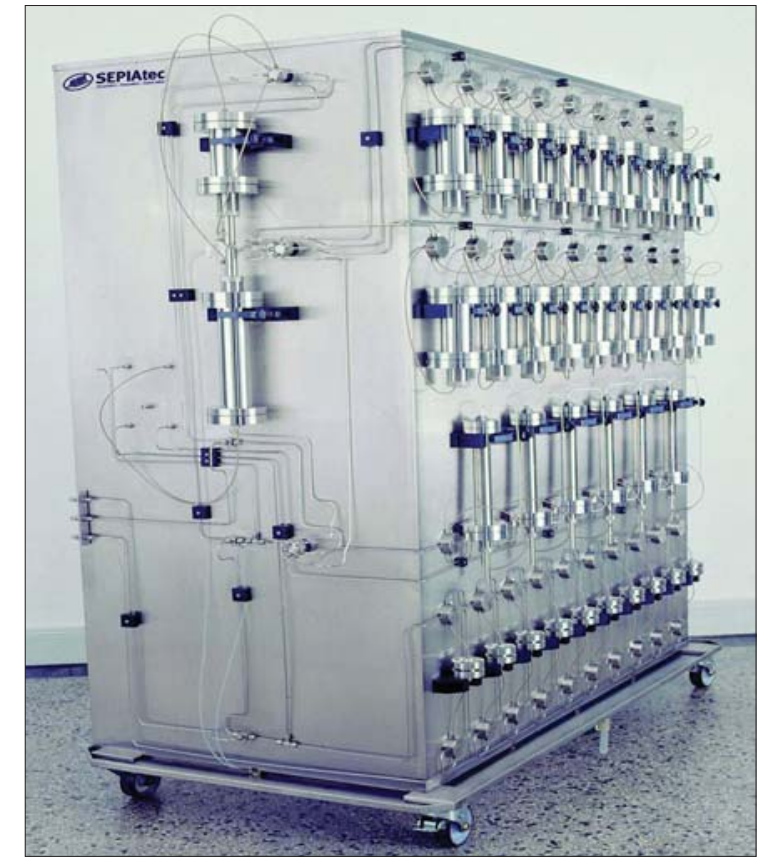

Figure 1: View of sepbox 
reproducible process one extract can be completely separated per day. The pure individual components are soluted in suitable solvents and can be collected in microtiter plates or vials. ${ }^{[1]}$ Sepbox uses alternative reverse phase column and SPE techniques. It can separate $5 \mathrm{~g}$ of the extract into approximately 300 fractions in organic solvent in $24 \mathrm{~h}$. Simulated moving bed chromatography system uses countercurrent solid-liquid extraction method. ${ }^{[2]}$

\section{PURIFICATION AND ISOLATION USING SEPBOX}

The purification and isolation processes are important for natural products. Screening systems for the identification of new bioactive compounds have a high throughput capacity and a successful lead identification is above all determined by the source material used. For further drug development, a compound in sufficient amount and purity is of crucial importance. The bioassay-guided fractionation procedure used to identify bioactive natural products is often perceived as rate-limiting and resourceintensive was shown in Figures 2 and 3. However, the rapid improvement of instrumentation and robotics used to revolutionize other aspects of drug discovery can also be used to improve the speed of isolation and structure elucidation of natural products. An increase in the speed of bioassay-guided fractionation has been facilitated by a marked improvement in HPLC automation, mass spectroscopy (MS) and column technologies as well as a rapid turnaround of screening results provided by HTS. The advent of new probe technology and higher magnetic fields has led to a significant shortening in acquisition time for nuclear magnetic resonance (NMR) data and the structure elucidation of natural products

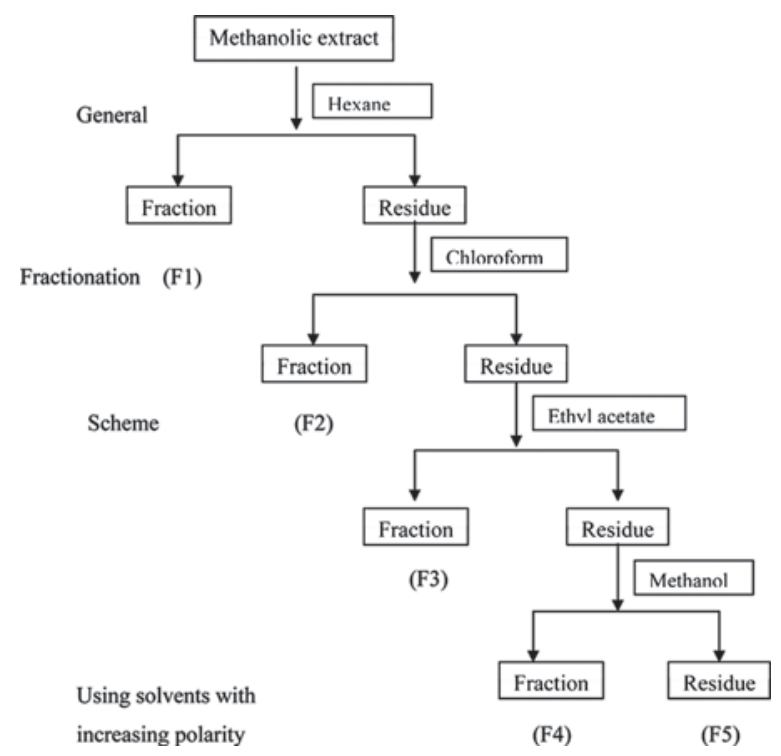

Figure 2: Scheme for fractionation of herbal material can be achieved routinely on amounts less than $1 \mathrm{mg}$. Automated chromatographic system (sepbox) is one of latest developments in the isolation and purification of bioactive compounds. ${ }^{[3]}$

\section{ADVANTAGES OF SEPBOX}

1. Sepbox produces high qualitative and reproducible results, has possibility to inject up to $5000 \mathrm{mg}$ of a natural extract and has the unique concept of totally automated preparative separation of natural products.

2. It has two-dimensional separation of an extract in up to 600 nearly pure substances within $24 \mathrm{~h}$ and fractions almost free of water.

3. Buffer of the second SPE step can immediately be processed and optimized column-switching through specific separating substances.

4. It is good manufacturing practice conform technique which has polar setup for intense polar substances and pipeline construction orbital welded.

5. It is quite imperative to initiate a symbiotic relationship with pharmaceutical industrial outfits and research and development institutions. ${ }^{[3,4]}$

\section{CLASSIFICATION OF SEPBOX}

Sepbox is classified as follows:
1. SEPBOX 2D
2. SEPBOX 1D
Sepbox 2D-5000
Sepbox 1D5000
Sepbox 2D-250
Sepbox 1D100
Sepbox 2D-100
Sepbox 1D-10

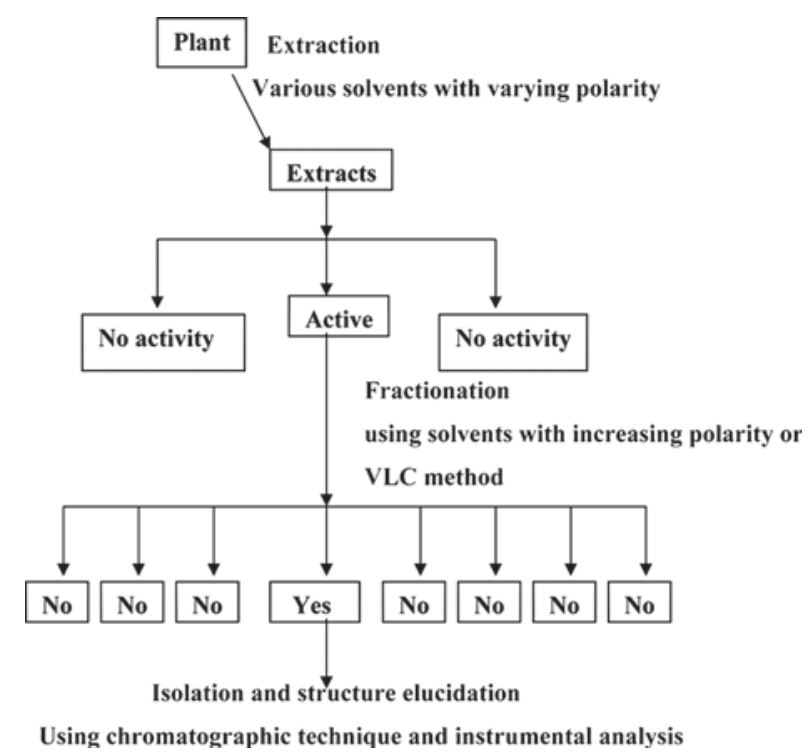

Figure 3: Bioassay-guided isolation of bioactive molecules 
SEPBOX 2D

Extracts of natural substances from $100 \mathrm{mg}$ to $5 \mathrm{~g}$ can be applied directly from microbiological, plant and animal material and separate highly polar substances using the Polar Setup. These units can detect almost all substances by using two detection systems (ultraviolet [UV] detection and an optional evaporative light scattering detection, ELSD). By these obtain quantities of individual chemical entities in a suitable solvent with and by which easily elucidate structures, screen different targets simultaneously and build a diverse library.

\section{SEPBOX 2D-5000}

Initially a reverse phase separation takes place and the obtained fractions are trapped on 18 SPE columns. The second separation step takes place on three alternative reverse phase columns depending on the polarity of the respective fraction.

Methods used: HPLC-SPE-HPLC-SPE

1. Up to $5000 \mathrm{mg}$ injection of crude natural extract.

2. Optional polar setup functions for highly polar substances.

3. Unique concept of a preparative fully automated separation of natural compounds.

4. In this instrument 6 HPLC and 18 SPE columns are placed in series.

5. Two-dimensional separation of an extract into up to 600 almost pure substances, within $24 \mathrm{~h}$.

6. Fractions almost free of water and buffer from the second SPE step can be processed immediately.

7. Optimized column switching using specific separating materials for maximum results.

\section{SEPBOX 2D-250/SEPBOX 2D-100}

Methods used: HPLC-SPE-HPLC

1. Optimized column switching.

2. Optional polar setup functions for highly polar substances.

3. Forty-eight hours of unattended operation when charged with six natural substance extracts.

4. Collection of fractions on microtiter plates which enables immediate use in HTS.

5. Second HPLC step done in parallel provides three times higher throughput compared to sepbox 2D-5000.

6. Downscaled versions of sepbox $2 \mathrm{D}-5000$ for separation of up to $250 \mathrm{mg}$ or up to $100 \mathrm{mg}$ of a crude natural extract.

7. Generation of sample material for multiple HTS assays under standardized separation conditions and at a high reproducibility.
SEPBOX 1D

The sepbox 1D family provides up to 50 extracts per day can be pre-fractionated with high reproducibility.

Samples taken from highly diluted and extremely complex mixtures in natural compound chemistry, combinatorial chemistry or when isolating impurities, byproducts and related substances of a synthesis can be concentrated easily.

\section{SEPBOX 1D-5000}

1. Injection of up to $5000 \mathrm{mg}$ of crude natural extract.

2. Preparative sample pre-fractionation of natural substances.

3. Individual setup for individual needs.

4. Fully automated patented HPLC-SPE coupling for high sample quality.

5. Fractions almost free of water and buffer obtained by the SPE step.

6. Efficient isolation and enrichment of impurities, byproducts and related substances of a synthesis by repeated injection permits their structural elucidation easily.

\section{SEPBOX 1D-100/SEPBOX 1D-10}

1. Injection of up to $100 \mathrm{mg}$ or up to $10 \mathrm{mg}$ of crude natural extract.

2. SPE/HPLC/SPE coupling for substance enrichment.

3. Fully automated system for pre-fractionation of extracts from natural substances.

4. Enrichment of highly diluted samples using an SPE injection module.

5. Parallelization option can multiply throughput. ${ }^{[4,5]}$

\section{PRINCIPLE OF SEPBOX}

Sepbox is a fully automated chromatographic instrument having dual combination of HPLC and SPE which permits enrichment and isolation of highly diluted compounds arranged as:

HPLC (separation) 》SPE (enrichment) » HPLC (separation) 》SPE (enrichment) 》 fractions shown in Figure 4. The sepbox combines the tools of sample preparation-reverse phase HPLC and SPE using standardized methods represents a novel solution for these difficulties. The separation conditions of the second HPLC step can be adjusted according to the polarity of the pre-fractions. ${ }^{[3]}$ 


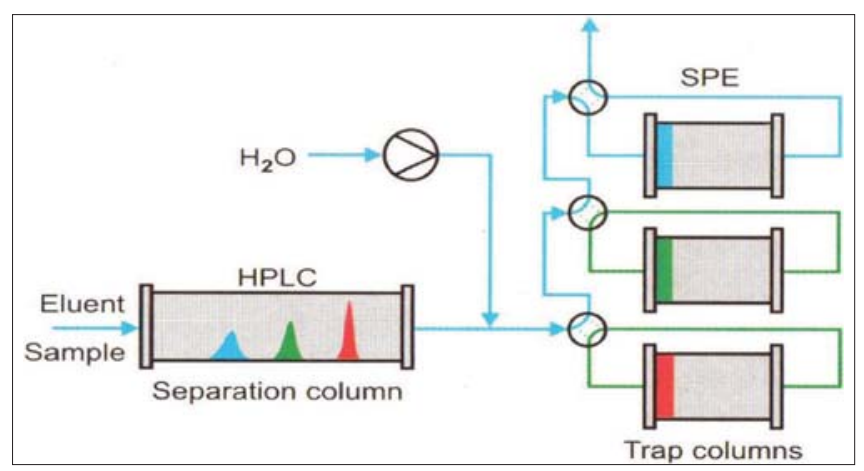

Figure 4: Principle of sepbox

\section{INSTRUMENTATION OF SEPBOX}

The Sepbox assembly consists of injection column, seven HPLC columns, 28 SPE columns (Trap column), four pumps, more than 50 valves, detectors (two UV and one ELSD), fraction collector $(6$ trays $\times 96$ vials $=576$ ), fractions at a time in a single run. ${ }^{[3]}$

\section{MODE OF OPERATION}

Reverse phase high-performance liquid chromatography

In reverse phase HPLC, the mixture to be separated is added to a mobile phase (mixture of methanol and water) which is forced through a column containing a stationary phase (typically chemically modified silica). The separation of the mixture is based on the difference in strength of the interaction of the components of the sample with the mobile phase and the stationary phase. Some components of the mixture will be strongly retained by the stationary phase and have a relatively weak interaction with the mobile phase; these compounds will exhibit a long retention time (that is, they are slowly eluted from the column). Other components will be weakly retained by the column and have a stronger interaction with the mobile phase; these compounds will exhibit a short retention time (that is, they are rapidly eluted from the column). To detect the elution of components from the column, some physical property of the mobile phase is monitored. The sepbox is supplied with a UV detector and an ELSD. The UV detector records the change in UV absorption when a compound is passing through its flow cell. The ELSD detects the change in light scattering of the mobile phase caused by a compound.

\section{Solid phase extraction on reverse phase}

Solid phase extraction (SPE) has been used for quite some time to collect, accumulate and isolate compounds in high dilution. The sepbox uses reversed phase extraction technology. In reversed phase extraction the column is packed with a non-polar adsorbent. The polarity of the mobile phase can be increased by addition of water, so that the dissolved non-polar compounds are adsorbed on the non-polar stationary phase. The thin layer adsorbed compound on the SPE column can be rinsed with water to remove buffer and other additives. Afterwards the compound can be desorbed with an organic solution.

\section{Solvent delivery system}

In liquid chromatography there are generally two modes of delivery of the mobile phase: The isocratic and the gradient mode. In the isocratic mode, the composition of the mobile phase remains constant during the separation. One pump is required for isocratic operation. In the gradient mode, the composition of the mobile phase is changed during the separation. A software program can conveniently generate the required gradient. The software is used to generate the gradient program. In general there are three different ways in which pumps can be employed to generate a gradient.

1. A low-pressure gradient uses one pump to deliver the desired mobile phase. A proportioning valve on the low-pressure side of the pump is used to generate the mobile phase.

2. A step gradient uses in general one pump and additional pump inlets to generate the mobile phase. The composition of the mobile phase is changed by opening one inlet valve and closing another inlet.

3. A high-pressure gradient uses two pumps to generate the mobile phase.

The pressurized solvent from the pumps is mixed before the sample is injected. Usually, the flow of one pump is kept constant while the flow of the other pump is changed as controlled by the computer. An advantage of the high-pressure gradient is that the change in composition of the mobile phase can be controlled more precisely than with a low-pressure gradient. The sepbox is therefore equipped with two pumps (Pump A and Pump B) to deliver the mobile phase. This allows the software to control the pumps in the isocratic mode and in the high-pressure gradient mode. The pumps can provide linear, convex, concave as well as step gradients. Pump A in general provides aqueous solvents like buffers and water while Pump B delivers the organic solvent, e.g. methanol and acetonitrile. Pumps A and B are each equipped with four valves is usually open while the other three are closed in normal operation. The open valves determine the delivery of solvents or solutions (e.g. buffer solutions) can be added when desired during the separation process. 


\section{Solid phase extraction unit}

After the UV detector (UV1) water is added (Pump C) to the mobile phase via high-pressure 3 -port-2 positions valve and the T-manifold to increase the polarity. This facilitates the absorption of the non-polar compounds on then non-polar compounds adsorption material in the SPE-columns (F1 to F18). The mobile phase is transported from the T-manifold to the 18 SPE columns (F1 to F18) via 6-port-2 positions-valve and $2 \times 3$-port- 2 positions valve (fraction valve). All $18 \mathrm{SPE}$ columns are linked via pneumatically driven 4-port-2-position valves (fraction 1.1 to 1.18). This allows each time-controlled fraction of the sample to be trapped on its respective SPE column.

\section{The fraction collection}

The sepbox fraction collector has six trays with 96 glass vials each. The fraction capacity is 572 fractions each with a volume of up $45 \mathrm{ml}$.

\section{Solvents}

Typical solvent amounts needed using default sepbox control methods.

Total solvent: 370 liters

Water : 240 liters

Acetonitrile : 72 liters

Methanol : 32 liters

Acetone : 27 liters

Packing of injection column

One to five $g$ defatted dried extract is dissolved in an appropriate quantity of methanol. The solution is filtered through a $0.22 \mu$ filter to remove any particulates that may be present. Next, three times the amount of reversed phase material is added (i.e. $2 \mathrm{~g}$ of extract to $6 \mathrm{~g}$ of reversed material). For a better distribution of the extract the mixture is completely dried in a rotary evaporator. If the material is not trickling (still building lumps) further reversed phase material has to be again mixed methanol and finally dried on a rotary evaporator. The injection column can now be filled with the help of the self-packing device. ${ }^{[3,4]}$

\section{APPLICATION OF SEPBOX}

1. Isolation and identification of bioactive compounds The root extract of $H$. rhamnoides (sea buckthorn) belongs to the genus hippophae (Family: Elaeagnaceae), is used as a medicinal plant in Tibet and is also native to Europe and Asia. The hippophae genus has several species but in India predominantly only three species, i.e. H. rbamnoides, H. salicifolia and H. Tibetan grow at the altitude of $2500-4500 \mathrm{~m}$. has been subjected to sepbox for the fractionation. First the sample is introduced via an injection column and separated at separation column I using a high-pressure gradient. The polarity of the eluant from separation column I is increased by the addition of water to such an extent that the fractions eluted from separation column I are adsorbed onto the 18 trap columns I. These trapped fractions are then eluted through the separation columns II where final separation is completed. Finally, the isolated and purified compound by sepbox is characterized by chromatographic and spectroscopic techniques such as infrared, NMR and MS. ${ }^{[3,6,7]}$

2. The antimalarial activity of Gomphostemma crinitum leaf extracts $5 \mathrm{~g}$ of crude extract was taken for fractionation by sepbox-2D 2000 automatic chromatographic system. ${ }^{[8,9]}$

3. Evaluating the effects of Erythroxylum cuneatum forma Cuneatum (Family: Erythroxylaceae) in morphineaddicted rats using sepbox 2D-250 to separate and fractionate crude extract using integrated HPLC-SPE system. The cytotoxicity effects of these extracts and their fractions were tested against vero cells a normal green monkey kidney cell-lines and the viability of the cells was assayed using sulphodaramine B assay. ${ }^{[10]}$

\section{CONCLUSION}

The natural medicines are attracting renewed attention. This is encouraging from both practical and scientific viewpoints their efficacy has often been proven over the centuries. However, to understand from nature is even more complex than mechanistic clarification of a single bioactive factor. This is because unfractioned or partly fractioned extracts are used, often containing mixtures of materials and in many cases synergism is most likely playing an important role. Improvements in instrumentation, robotics and bioassay technology have increased the speed of bioassay-guided isolation and structure elucidation of natural products considerably and these improvements have allowed natural products' research to be more competitive with synthetic compound screening.

Sepbox is a more efficient and fast technology which has increased the speed of bioassay-guided isolation and structure elucidation of natural products. 


\section{REFERENCES}

1. Available from: http://www.zinsserna.com/sepbox.htm. [last cited on 2010 Sep 1].

2. The National chemical laboratory (NCL). Combinatorial techniques in drug discovery. Pune: CSIR; 2003.

3. Sharma RK, Arora R. Herbal drugs: A twenty first century perspective. $1^{\text {st }}$ ed. New Delhi: Jaypee Brothers and Medical Publishers; 2006. p. 156-61.

4. Available from: http://www.chemito.net/uploads/product/pdf/17_pdf. pdf. [last cited on 2010 Sep 1].

5. Agrawal SS. Paridhavi M. Herbal drug technology. $1^{\text {st }}$ ed. Hyderabad: Universities Press; 2007. p. 338-41

6. Swaroop A, Sinha AK, Sharma RK, Kumar JK. A novel from berries of Hippophae rhamnoides. Chem Pharm Bull 2005;53:1021-4.
7. Singh V, Gupta RK. Seminar on Sea buckthorn. A resource for environment, Health and Economy; Directorate of life sciences (DRDO), New Delhi; 2004.

8. Cragg GM, Newman DJ, Snader KM. Natural products in drug discovery and development. J Nat Prod 1997;60:52-60.

9. Handa SS, Mangal AK, Mundkinajeddu D. Indian herbal pharmacopeiaregional research lab; Jammu and Indian Drug Manufacturer's Association; 1998. p. 109.

10. Available from: Http://info.frim.gov.my/cfdocs/infocenter/Highlight/ IRPA_2007/DrIlhamAdenan.pdf. [last cited on 2010 Sep 1].

How to cite this article: Bhandari M, Bhandari A, Bhandari A. Sepbox technique in natural products. J Young Pharmacists 2011;3:226-31.

Source of Support: Nil, Conflict of Interest: None declared.

\section{Author Help: Reference checking facility}

The manuscript system (www.journalonweb.com) allows the authors to check and verify the accuracy and style of references. The tool checks the references with PubMed as per a predefined style. Authors are encouraged to use this facility, before submitting articles to the journal.

- The style as well as bibliographic elements should be $100 \%$ accurate, to help get the references verified from the system. Even a single spelling error or addition of issue number/month of publication will lead to an error when verifying the reference.

- Example of a correct style Sheahan P, O'leary G, Lee G, Fitzgibbon J. Cystic cervical metastases: Incidence and diagnosis using fine needle aspiration biopsy. Otolaryngol Head Neck Surg 2002;127:294-8.

- Only the references from journals indexed in PubMed will be checked.

- Enter each reference in new line, without a serial number.

- Add up to a maximum of 15 references at a time.

- If the reference is correct for its bibliographic elements and punctuations, it will be shown as CORRECT and a link to the correct article in PubMed will be given.

- If any of the bibliographic elements are missing, incorrect or extra (such as issue number), it will be shown as INCORRECT and link to possible articles in PubMed will be given. 